\title{
Novel Technique for Replication of Natural Palatal Anatomy onto the Maxillary Permanent Denture Base within an Even Thickness
}

\author{
${ }^{1} \mathrm{C}$ Guna Sekar, ${ }^{2}$ Chitraputhrapillai Sabarigrinathan, ${ }^{3}$ Krishnamoorthy Vinayagavel, ${ }^{4}$ Karthikeyan Ramkumar \\ ${ }^{5}$ Muthuveeraganapathy Dhanraj
}

\begin{abstract}
Aim: A technique to reproduce the exact natural anatomy of the palatal surface, known as "anatomical contour" in even thickness onto the maxillary permanent denture base.
\end{abstract}

Background: In constructing oral prostheses, dentists are primarily concerned with replacing structural losses. However, we are necessarily adding to regions having no structural loss, e.g., the hard palate region. When these structures are covered by a denture, the proprioceptive feedback mechanism is changed and, naturally, dentures affect phonetics, which causes a temporary change in the articulation of speech sounds.

Technique: A metal ring plate in standardized different thicknesses $(1.5,2$, and $2.5 \mathrm{~mm}$ ), which adapt to dental flask lower base, is made and used in the routine fabrication method of denture base processing.

Conclusion: The quick and easy methods of replicated entire individualized natural anatomical palatal contour, in various even thicknesses in the permanent maxillary denture base, including the incisive papilla, palatine suture, and palatal rugae, can be employed to newly fabricate maxillary complete dentures.

Clinical significance: This is a comprehensive approach to utilize the collective factors of suggested methods, techniques, and conclusion by various authors for speech improvement with maxillary denture.

Keywords: Denture base, Denture base thickness, Novel technique, Palatal contour, Replication.

How to cite this article: Sekar CG, Sabarigrinathan C, Vinayagavel K, Ramkumar K, Dhanraj M. Novel Technique

\footnotetext{
${ }^{1}$ Research Scholar, Head and Staff Surgeon, ${ }^{2} \mathrm{Head}$, Professor and Guide, ${ }^{3,5}$ Professor, ${ }^{4}$ Associate Professor

${ }^{1}$ Department of Dentistry, ESIC Medical College and Postgraduate Institute of Medical Sciences and Research, Chennai, Tamil Nadu, India

2,3 Department of Prosthodontics, Tamil Nadu Government Dental College, Chennai, Tamil Nadu, India

${ }^{4}$ Department of Dental Surgery, Stanley Medical College Chennai, Tamil Nadu, India

${ }^{5}$ Department of Prosthodontics, Saveetha Dental College Chennai, Tamil Nadu, India

Corresponding Author: C Guna Sekar, Research Scholar Head and Staff Surgeon, Department of Dentistry ESIC Medical College and Postgraduate Institute of Medical Sciences and Research, Chennai, Tamil Nadu, India, Phone: +914424713368 e-mail: cgsekar63@gmail.com
}

for Replication of Natural Palatal Anatomy onto the Maxillary Permanent Denture Base within an Even Thickness. Int J Prosthodont Restor Dent 2017;7(3):92-96.

Source of support: Nil

Conflict of interest: None

\section{INTRODUCTION}

A major portion of speech articulation takes place within the oral cavity, and any alteration of structures therein will adversely affect speech proportionate to the location and magnitude of the alteration. ${ }^{1}$

Failure to contour the palate to accommodate normal tongue contact usually results in poor speech for a period of several days or several weeks, depending upon the aptitude of the patient and the discrepancy in palatal contour. However, speech articulation takes place when an approximation or movement of the articulators constricts, impedes, or diverts the airstream to produce a single sound. Contour palate for proper tongue contact is difficult, time-consuming, or expensive. However, it does require some knowledge of the fundamentals of phonetics and a precise knowledge of the normal tongue contact areas for speech articulation.

In constructing oral prostheses, dentists are primarily concerned with replacing structural losses. However, we are necessarily adding to regions having no structural loss, for example, the hard palate region. The anterior third of the palate has been referred to as the playground of the tongue. ${ }^{2}$ When these structures are covered by a denture, the proprioceptive feedback mechanism is changed and, naturally, dentures affect phonetics, which causes a temporary change in the articulation of speech sounds. The adaptability of the tongue progresses slowly, which is better in younger patients than older ones. Tanaka ${ }^{3}$ concluded that palatal vaults of maxillary dentures should be shaped like natural ones to facilitate correct pronunciation. He also reported that quality of speech sound production improved within the first week of insertion of new denture. The slight bulging of the denture base palatal to the anterior teeth probably helps in improved pronunciation of /s / and /sh/ sounds. ${ }^{1}$

Consonants are articulated speech sounds, and all require articulation to impede, constrict, divert, or stop 
the airstream at the proper place and time in the oral and nasal cavities to produce the desired sound.

The tongue is the principle articulator for speech. To establish the normal tongue contact with the palate of the denture, one must know the normal tongue-palate contact. The loss of teeth and supporting structures alters the main articulatory cavity and produces a marked impact on this speech pattern.

The proper palatal contour is very much essential to have proper /s / pronunciation for that matter, and the fact is that the palate can be contoured to accommodate phonetics for any sensible tooth arrangement. John M Palmer ${ }^{4}$ quoted the earlier authorities' suggestions of nearly exact replication of the natural anatomy into the denture base having supported the notion of duplicating the palatal rugae. This has not been verified, nor has it become general practice, for speech purposes.

The purpose of this article is to replicate quick and easy methods of adding entire individualized natural anatomical palatal contour, in even thickness onto the permanent denture base, including the incisive papilla, palatine suture, and palatal rugae to newly fabricated complete dentures.

\section{TECHNIQUE}

Primary impression was made with impression compound (Y-DENTS, MDM Corporation, New Delhi, India), and primary casts were made with type II gypsum (Plaster of Paris, Ramaraju Surgical Cotton Mills Ltd., Sankarankovil, Tamil Nadu, India). Custom trays were fabricated with chemically activated denture base resin (DPI-RR Cold Cure, Dental Product of India, Mumbai, India) with $1.5-\mathrm{mm}$ wax spacer. Trays were trimmed and tried in the patient's mouth to check the extension. Then, the border molding was done with tracing compound (DPI Pinnacle Tracing Sticks, DPI Mumbai, India) and secondary impressions were made with mucostatic impression material (light body rubber base impression material, 3M ESPE Express, 3M India Pvt Ltd., Bengaluru, India). Master casts were poured with type III dental stone (Gem Stone; Shruti Products, India).

\section{DIFFERENT FROM CONVENTIONAL METHODS}

\section{Processing Methods for Fabrication of Modified Palatal Surface Contour}

- Duplicate maxillary master casts were prepared using duplicating silicone (Unisil-flow, Delta, made in Germany) to achieve similar surface structure (Fig. 1).

- To reproduce the exact natural anatomy of the palatal surface, known as "anatomical contour" in even thickness, without losing the original surface details of the impression, the modified permanent denture base was prepared, by using a dental stone master cast, placed in the bottom half of the dental flask and the cast, and was painted with separating medium (Fig. 2). The sloping was given in the investing material, between the edge of the cast and the rim of the flask. The outer rim of the flask was kept free of the dental stone.

- The lab putty material (Unisil-Putty, Delta, made in Germany) was mixed according to manufacturer's instruction and a layer of this mixed lab putty was placed over the master cast in the lower half of the flask (Fig. 3). Before placing the putty material, undercuts in the master cast (sulcus to ridge region) were blocked out with modeling wax or lab putty material to facilitate the easy separation of the flask. The separating medium was applied, and the upper half of the flask was placed in a position in close contact with the lower half, and the dental stone was poured to fill up the flask along with gentle tapping to avoid air bubbles or voids. The lid was closed, the flask

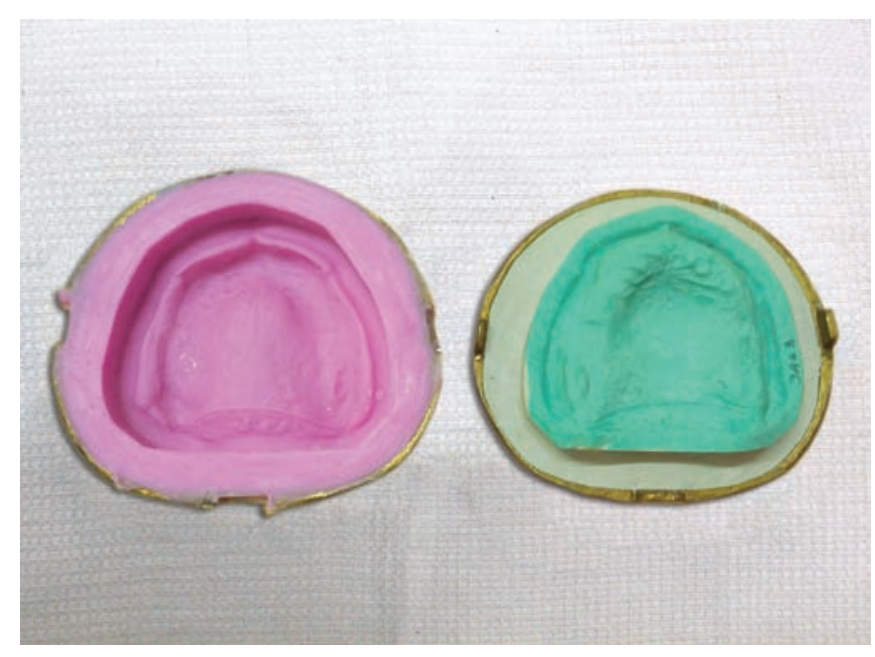

Fig. 1: Duplicating master cast

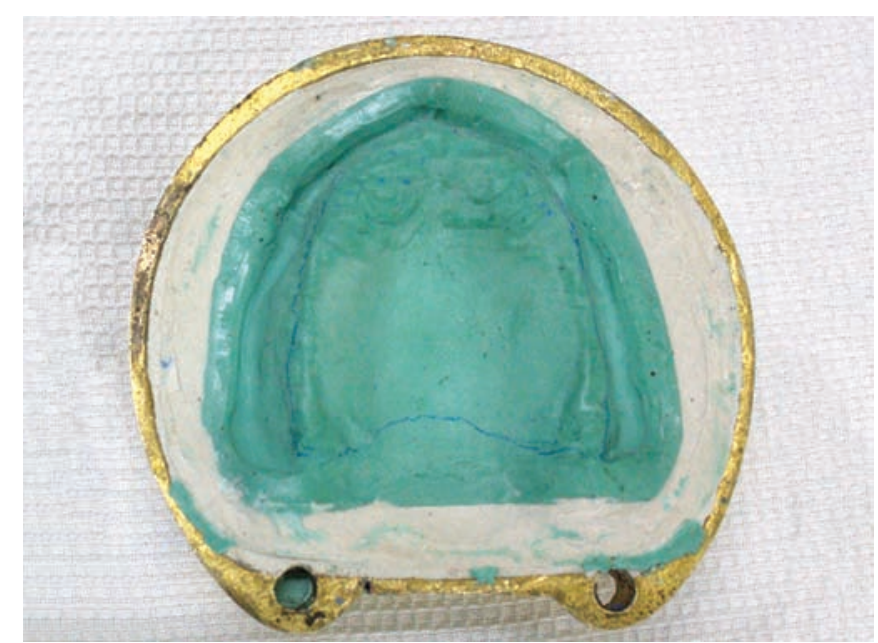

Fig. 2: Master cast positioned on the lower base of the dental flask 


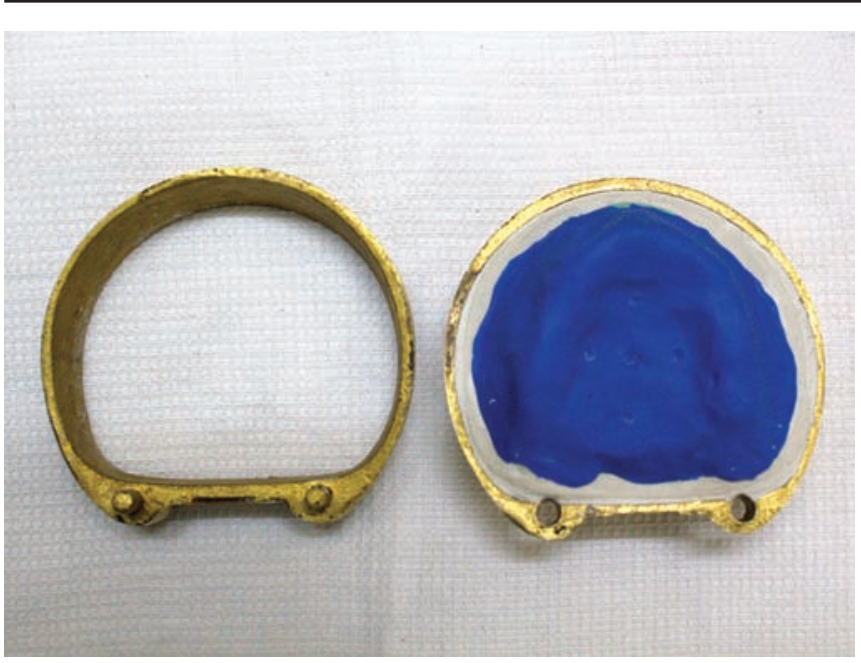

Fig. 3: Lab putty over the master cast

is tightened by clamping, and the dental stone was allowed to set completely before opening the flask.

- The lower half of the flask was separated without distorting the cast, the modeling wax or lab putty material covering the undercuts was removed, the lab putty index replicating the natural anatomical contour of the palate was held up in the upper half of the flask (Fig. 4).

- Separating medium was applied over the master cast in the lower half of the flask; similarly the separating medium was applied over the upper half of the flask adjacent to the putty index.

- Denture Resin (Lucitone $199^{\circledR}$, made in Germany), an acrylic base material, was mixed with correct powder and liquid ratio in accordance with the manufacturer's directions. The resin dough was rolled and flattened into a $1 / 4$-inch thickness $(6 \mathrm{~mm})$ and pieces are cut to approximate length of the flanges and the size of the palate.

- Then, indigenously designed metal ring plate (Fig. 5) with a standardized thickness of $1.5,2$, and $2.5 \mathrm{~mm}$

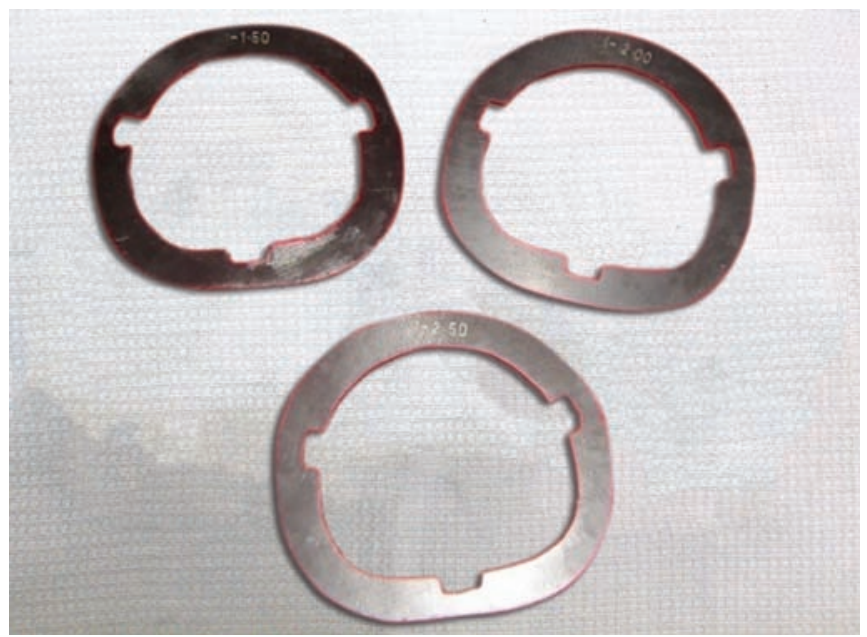

Fig. 5: Metal ring designed to standardize uniform thickness of upper permanent denture base

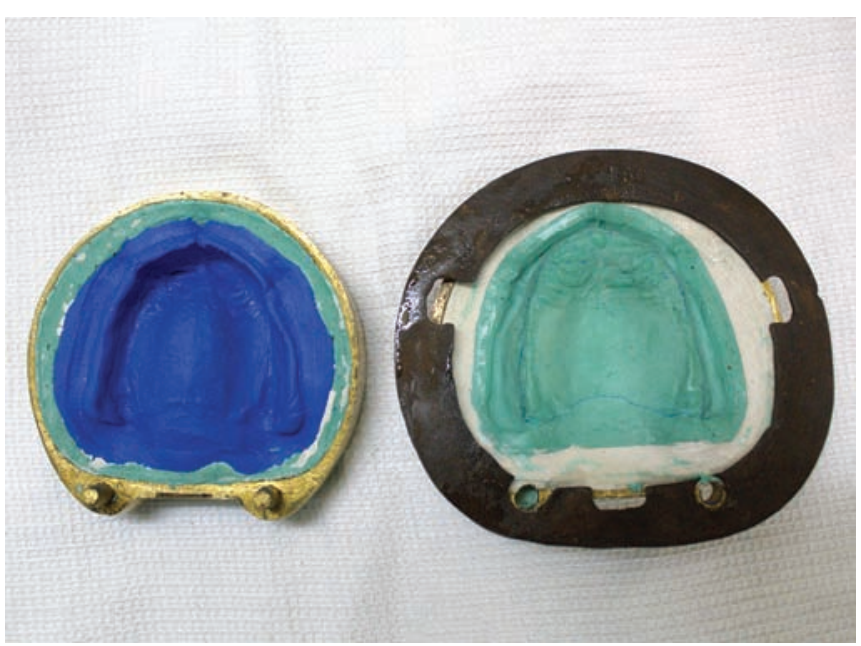

Fig. 4: Index palatal contour and metal ring placement

was used. We chose one that adapted with the rim of the lower half of the flask as shown in Figure 4, and the upper half of the flask was placed over the metal ring, and the flask was placed in the press.

- The flask was closed at the press with a cellophane sheet placed between the two halves and the flask was tightened in the press until the two halves were in close approximation. Then the flask was opened, excess resin was cut away precisely at the border, and additional resin was added if any place was found deficient. The cellophane sheet was removed. Then the flask was closed completely and transferred to the spring clamp to make it tighter.

- The curing procedure was carried out and processed according to the recommend temperature given by the manufacturer (long curing cycle). After completion of curing, the flask was removed and bench cooled. After deflasking, the denture base was retrieved (Fig. 6) from the stone cast and the permanent denture base trimmed for the excess and shaped and polished (Fig. 7).

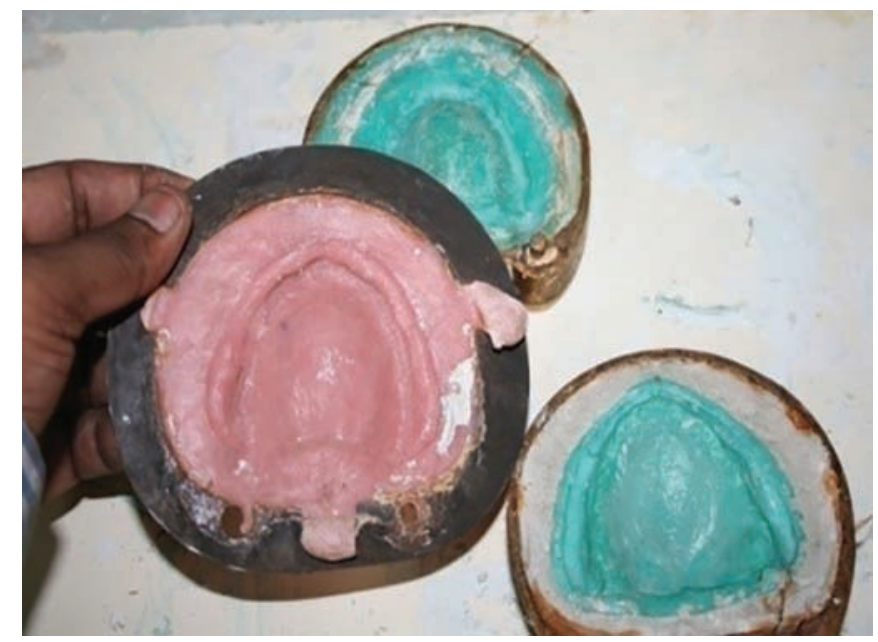

Fig. 6: Deflasking denture base 


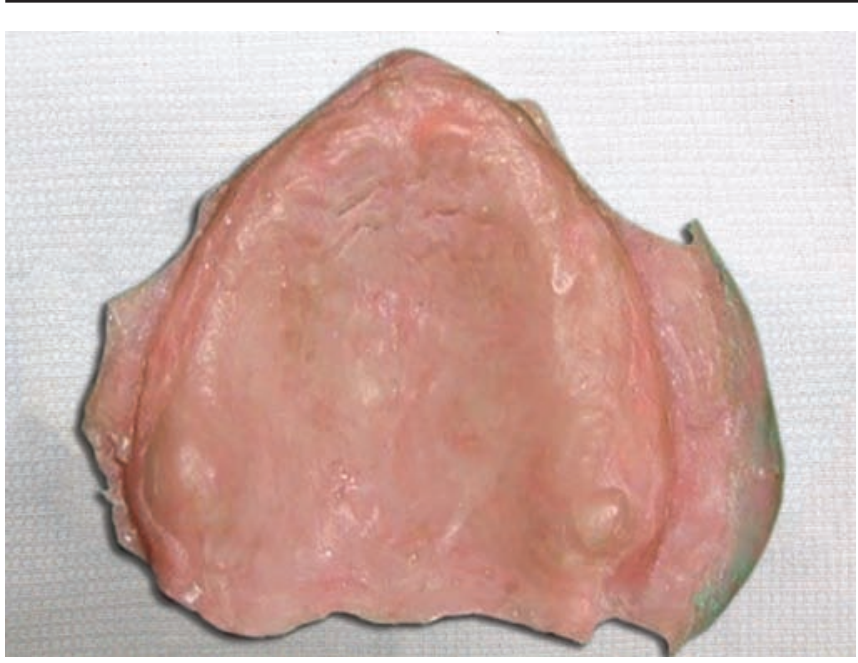

Fig. 7: Replicated palatal contour on the permanent denture base in even thickness

- The processed permanent record base was achieved with even thickness and the exact surface details of the master cast on the polished palatal surface were obtained. If required, the processing procedure was repeated with the other ring sizes for the remaining two different even thicknesses to achieve similar palatal contours.

The occlusal rim was fabricated over the modified permanent denture base. The jaw relation recording procedure was carried out on the occlusal rim, and with correctly established vertical and horizontal relationships, it was transferred to the articulator for teeth arrangement (Premadent, Super Dental Products, Delhi, India).

After completing the trial and verification of jaw relation, the wax trial dentures were flasked and processing with heat cure acrylic resin was performed in the usual manner. Shrinkage was not affected by the thickness of the acrylic resin, in the presence or absence of undercuts or two curing cycles. ${ }^{5}$ The flask was removed, bench cooled, and deflasking was done. The dentures were trimmed and polished, before they were remounted in the articulator to check the occlusion.

\section{DISCUSSION}

There are many methods in duplicating the rugae. ${ }^{6-12}$ All these techniques are simulated, added, engraved, fabricated, transformed, or customized ${ }^{13}$ onto the surface of the plate. None of these techniques has achieved exact replacement of the natural anatomy in an even thickness. This technique is superior because of fulfilling the exact replication of the individualized natural palatal anatomy ${ }^{4}$ in even thickness ${ }^{14}$ of the entire palatal surface including the rugae region and the incisive papilla region.

All the techniques related to speech improvement in complete dentures were made under a specific aspect for the better quality of speech. In this present method, many authors' aspects adopted collectively to improve speech function with a complete denture.
Earlier authors (Bloomer and Luchsinger and Arnold $)^{15,16}$ who are suggesting nearly exact replication of the natural anatomy onto the denture base have supported the notion of duplicating the palatal rugae. This has neither been verified nor has it become general practice for speech purposes. This has been verified with this technique in general practice.

Specifically, this technique altogether increases the tactile surface area in the anterior region of tongue-todenture contact, provides good functional room for the tongue, and the direct visualization of the papilla on the palatal surface of the permanent denture base provides a better guide for positioning the anterior teeth. This is a universal and easy method for refining the speech function with the maxillary complete denture.

\section{CONCLUSION}

The advantages of the described procedures are that they can easily and quickly be accomplished by the dentist or the laboratory technician. This simple method of replicating an entire individualized natural anatomical palatal contour in various even thicknesses onto the permanent maxillary denture base, including the incisive papilla, palatine suture, and palatal rugae, helps to newly fabricate complete dentures for the enunciation of normal speech immediately after the insertion. This, thereby, eliminates or considerably reduces postinsertion practice period, and can accomplish speech improvement.

\section{CLINICAL SIGNIFICANCE}

This is a comprehensive approach for improving the speech sounds production using the collective factors that are involved in speech articulation on complete denture wearer.

\section{ACKNOWLEDGMENT}

The authors readily acknowledge their indebtedness to retired Professor, Dr C Thulasingam, MDS, Tamil Nadu Government Dental College, Chennai, Tamil Nadu, India.

\section{REFERENCES}

1. Goyal BK, Greenstein P. Functional contouring of the palatal vault for improving speech with complete dentures. J Prosthet Dent 1982 Dec;48(6):640-646.

2. Martone AL, BlackJW. An approach to prosthodontics through speech science: part V. Speech science research of prosthodontic significance. J Prosthet Dent 1962 Aug;12(4):629-636.

3. Tanaka H. Speech patterns of edentulous patients and morphology of the palate in relation to phonetics. J Prosthet Dent 1973 Jan;29(1):16-28.

4. Palmer JM. Structural changes for speech improvement in complete upper denture fabrication. J Prosthet Dent 1979 May;41(5):507-510. 
5. Lechner SK, Lautenschlager EP. Processing changes in maxillary complete dentures. J Prosthet Dent 1984 Jul;52(1): 20-24.

6. Kaufman C, Cardash HS. A method of restoring the palatal form in a complete maxillary denture. J Prosthet Dent 1984 Jan;51(1):126-128.

7. Gitto CA, Esposito SJ, Draper JM. A simple method of adding palatal rugae to a complete denture. J Prosthet Dent 1999 Feb;81(2):237-239.

8. Kong HJ, Hansen CA. Customizing palatal contours of a denture to improve speech intelligibility. J Prosthet Dent 2008 Mar;99(3):243-248.

9. Abdelhameed HM, El-Sayed ME. Effect of different techniques for palatal denture base configuration on speech quality in complete denture wearers. Med J Cairo Univ 2014 Sep; 82(1):657.

10. Sanjay VB, Priti S, Sadekh A. Reproducing functional palatal contours in complete dentures to improve speech-a case report. J Indian Dent Assoc 2012;6(2):111-114
11. Vijayaraghavan V, Chandni P. A simple method for palatal rugae carving in complete dentures. J Indian Prosthodont Soc 2013 Jun;13(2):137-138

12. Neelakantan A, Dhaded S. Rugae duplication-different techniques of customizing palatal rugae in maxillary complete denture to enhance phonetics. Karnataka Prosthodont J 2016 Jun;1(1).

13. Naqvi S, Dange SP, Khalikar SA. A simple method for patients palatal rugae duplication in complete dentures. Int J Prosthodont Restor Dent 2014 Apr;4(2):46.

14. Reeson MG, Jepson NJ. Achieving an even thickness in heat-polymerized permanent acrylic resin denture bases for complete dentures. J Prosthet Dent 1999 Sep;82(3):359-361.

15. Bloomer HH. Speech defects associated with dental malocclusions and related abnormalities. In: LE Travis, editor. Handbook of speech pathology and audiology. New York: Appleton-Century-Crofts; 1971 p. 715-765.

16. Luchsinger, R., Arnold, G.E. Voice-Speech-Language. in: Wadsworth Publishing Co., Belmont, Calif.; 1965:650. 